\title{
Structure and Sedimentation Kinetics of Dense Suspensions of Fibroblast Cells
}

\author{
Arbnor Zenunia ${ }^{a}$, Chi Zhanga, Laetitia Haeni' ${ }^{b}$, Barbara Rothen-Rutishauser ${ }^{\star b}$, and Frank Scheffold ${ }^{\star a}$
}

\begin{abstract}
We investigate the structure and the dynamics of dense suspensions of NIH $3 \mathrm{~T} 3$ fibroblast cells. Using two-photon microscopy we obtain three dimensional (3D) images from which the size and the packing structure of the dense cell suspensions can be extracted. In addition, we analyse the global time-dependent behaviour of the suspensions by time-lapse measurements of cell sedimentation. Since cell adhesion is a non-equilibrium living process the interplay can be influenced by cell viability interfering with cell-cell interactions.
\end{abstract}

Keywords: Colloidal suspensions · Fibroblast cells · Two-photon microscopy

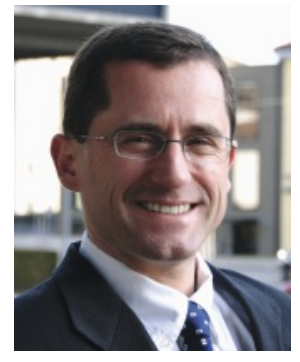

Frank Scheffold studied at the University of Konstanz (Germany) and did his diploma work with Prof. Jacob Klein at the Weizmann Institute of Science (Israel) on polymer blends. He received his $\mathrm{PhD}$ from the University of Konstanz (Germany) in 1998 for research on diffusive light scattering and colloidal dynamics, carried out at the Institute Charles Sadron (Strasbourg, France) and Konstanz with Prof. Georg Maret. After postdoctoral work with Prof. Peter Schurtenberger and a habilitation on 'Optical Probes in Soft Matter Physics' at the University of Fribourg he was appointed associated professor in 2004 and since 2009 he holds the chair 'Soft Matter and Photonics'. He has been a visiting fellow at UC Los Angeles (2009) and the University of Queensland, Brisbane (2014). His research focuses on the optics of complex dielectric media, dynamic light scattering and diffuse light propagation, the dynamics, aggregation and phase behaviour of colloidal systems and the fabrication and characterisation of soft materials. He currently serves as a member of the Swiss National Research Council and as the chairman of the board of the technology company LS Instruments AG, Fribourg. Prof. Scheffold leads Module 2 in the NCCR 'Bio-Inspired Materials'.

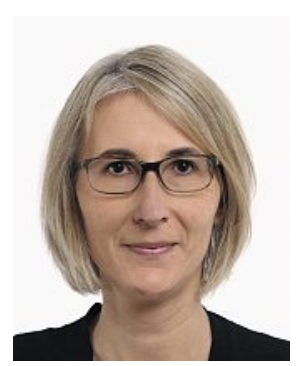

Barbara Rothen-Rutishauser received her $\mathrm{PhD}$ in 1996 in cell biology at the Swiss Federal Institute of Technology (ETH) in Zurich. From 1996 to 2000 she held a post-doctoral position in Biopharmacy at the Institute of Pharmaceutical Sciences at the ETH and in 2000 she joined Prof. Peter Gehr's research group at the University of Bern, Switzerland as a postdoc. After promotion to group leader in 2006 she completed her habilitation in cell biology in 2009. B. RothenRutishauser is an expert in the field of cell-nanoparticle interactions in the lung, with a special focus on 3D lung cell models and various microscopy techniques such as laser scanning and

\footnotetext{
${ }^{*}$ Correspondence: Prof. F. Scheffold ${ }^{a}$, Prof. B. Rothen-Rutishauser ${ }^{b}$ E-mail: frank.scheffold@unifr.ch, barbara.rothen@unifr.ch aDepartment of Physics, University of Fribourg, Chemin du Musée 3, $\mathrm{CH}-1700$ Fribourg

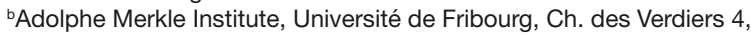
$\mathrm{CH}-1700$ Fribourg
}

transmission electron microscopy. Since 2011 she is the chair in BioNanomaterials at the Adolphe Merkle Institute, University of Fribourg, Switzerland, the position is shared equally with Prof. Alke Fink. The research group's activities stretch over many fields from material synthesis and characterization to biological responses and hazard assessment. Prof. Rothen-Rutishauser has published more than 220 peer-reviewed papers and is an associate editor of the journal Particle and Fibre Toxicology. Together with Prof. Alke Fink she co-leads Module 3 in the NCCR 'Bio-Inspired Materials' and she is the faculty delegate for equal opportunities.

\section{Introduction}

Particle suspensions at high concentrations can exhibit complex non-Newtonian fluid behaviour. ${ }^{[1]}$ If packed densely they can display a glass and a jamming transition leading to a material with a finite shear modulus due the repulsive restoring forces when the particles are compressed and deformed. Such behaviour is well known in the context of food, paint and pharmaceutical systems, i.e. for suspensions of randomly distributed hard or soft particles in a fluid medium. ${ }^{[2-6]}$ However, for suspensions of a biological nature, only a few studies exist. ${ }^{[4]}$ The main focus of these previous studies has been on the rheology of suspensions such as red blood cells, Chinese hamster ovary cells and rat aorta smooth muscle cells. ${ }^{[6,7]}$

In our work we are investigating the structure and dynamics of common living NIH 3T3 fibroblast cells established from mouse embryo cultures. The fibroblast cell type has been chosen because of their mesenchymal origin which implies that they are dependent on adhesion to either the extracellular matrix (ECM) or to other cells for survival. ${ }^{[4]}$ The cells were purchased by ATCC (American Type Culture Collection) and were kept in culture in Dulbecco's Modified Eagles medium (DMEM), a high glucose medium that contains $10 \%$ of Fetal Bovine Serum (FBS) and 1\% of Penicillin/Streptomycin as recommended by the provider. They acquire nearly spherical shapes when kept suspended with a cell diameter of about 15 micrometres, resembling particle suspensions with a size in between the colloidal and the granular range. What is new and interesting about these cell suspensions is that the 'particles' (cells) interact via their interface, which is sticky due to cell-surface bound protein receptors, and these attractive interactions are expected to depend on whether the cells are alive or dead.

Mesenchymal and other biological cells are often studied in culture using light microscopy with a lateral resolution in the sub-micrometre range. However, due to strong scattering and the 
need for optical sectioning, a conventional light microscope is not well suited for the study of dense cell suspensions in three dimensions. Thus, for many in vitro experiments, confocal or two-photon microscopy is used. Here we use the latter technique and to this end we first label the cells with Rhodamine-B isocyanate and then use a custom-made two-photon microscope for imaging. $\left[{ }^{[8,9]}\right.$ Compared to confocal microscopy the two-photon microscope offers the advantage of a substantial increase in accessible depth based on the principle of non-descanned detection of emitted light. ${ }^{[9,10]}$ The necessary condition for two-photon fluorescence excitation (in the UV) and emission (in the visible spectral range) is that two photons simultaneously excite a fluorescence molecule. We aim a tightly focused fs-pulsed near infrared beam on the sample using a Coherent Mira 900 Ti:Sapphire laser system and collect the green emitted light at a wavelength $\lambda \simeq 590 \mathrm{~nm}$ to obtain a 2D image section by scanning the beam in a plane (Fig. 1). By additionally scanning along the axial (z) direction we obtain a $z$-stack of images which can be rendered in a 3D view as shown in Fig. 1d. ${ }^{[11,12]}$ We perform 3D template-based particle tracking as described in ref. [12] and obtain the coordinates and the size of each cell. Based on this approach the size of the cells is determined to be approximately $11.5 \mu \mathrm{m}$.

\section{Radial Distribution Function}

From the 3D image (Fig. 1) we can determine the radial distribution function of the cell centre positions. The sample cuvette has a volume of $25 \mu \mathrm{l}$ with a height of $120 \mu \mathrm{m}$ and we use the entire volume for our analysis. Due to the relatively large size of the individual cells the total number of cell layers is limited to about eight. We can extract reliable measures of the radial distribution function $g(r)$ as shown in Fig. 1e.

The pair correlation functions for a liquid-like assembly of uniform colloidal particles (and atomic liquids) are well studied. ${ }^{[13]}$ In the absence of crystallization, the structure is isotropic and the pair correlation function reduces to the radial distribution $g(r)$. For suspensions with a finite size distribution and a polydispersity of more than 6-7\%[14] (standard deviation divided by the mean), like our cell suspensions, crystallization is always sup pressed and the dense suspensions remain disordered. ${ }^{[15]}$ For hard spheres or in the presence of only very weak attractions $g(r)$ is predominantly set by the steric excluded volume occupied by the particles, expressed by the space filling or volume fraction. For the case of stronger particle-particle attraction, phase separation can set in or non-equilibrium stable or meta-stable gels are formed. ${ }^{[16]}$ In our case we find that the measured $g(r)$, at volume fractions of 0.2 and above can be described by the wellknown Percus-Yevick theory for repulsive hard spheres ${ }^{[13,17]}$ (taking polydispersity into account as described in ref. [12]). The parameters we obtain for a best fit of $g(r)$ are also in quantitative agreement with the size and density determined directly from the image analysis (Fig. 1). The presence of short-range attractive forces, which would only weakly affect the $g(r)$ on the length scales studied by microscopy, cannot be easily resolved in our imaging experiments. To overcome these difficulties, it would be desirable to monitor the cell dynamics, i.e. their motion and thermal diffusion in space. Other 'living' biological suspensions such as bacteria display active propulsion and enhanced diffusion using their flagella. ${ }^{[18,19]}$ However, since our fibroblast cells are not actively driven, we expect a subtler influence of the biological activity on their dynamics. The thermal motion and the phase behaviour can be altered by the presence of 'living' interactions, i.e. sticky interactions and reorganization processes in the ECM.

In practice we find that the cell motion at lower number densities is masked by rapid sedimentation due to gravity. This is the dominant mechanism in our case, due to the large buoyancy mismatch and rather large cell size. It is thus difficult to study time-dependent properties such as cell motion and thermal diffusion. To overcome this problem, we study directly the sedimentation behaviour of living cells at higher densities where transient gel-like structures are formed and compare our observation to the well-studied behaviour of submicron sized colloids with attractive depletion interactions induced by adding non-absorbing polymers. ${ }^{[20]}$
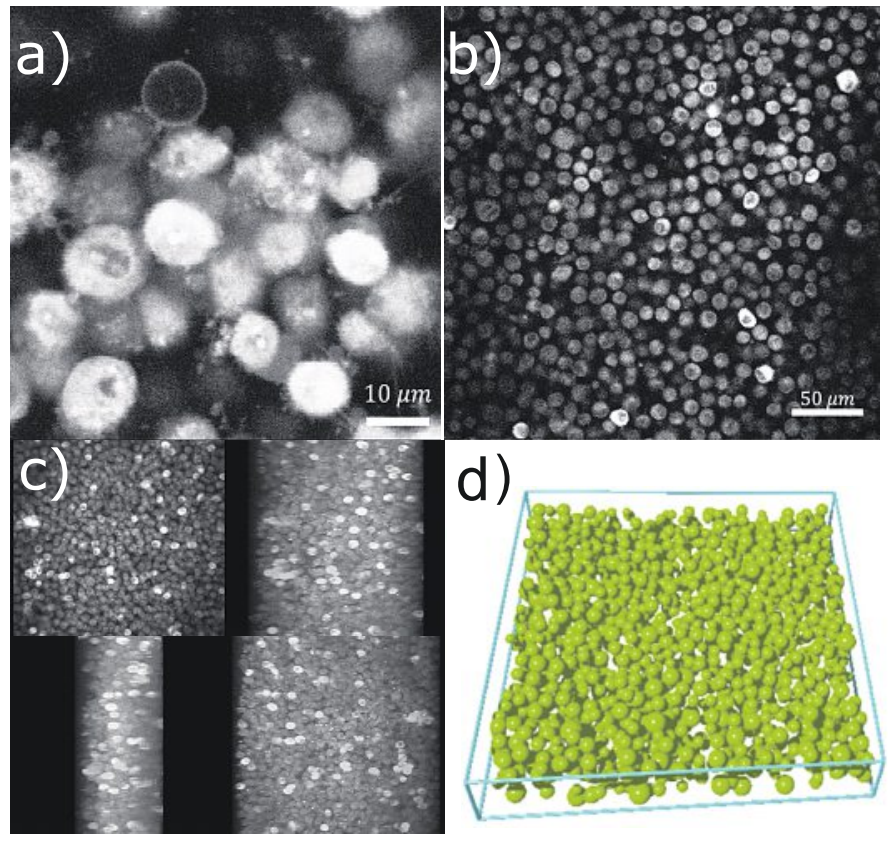

e)

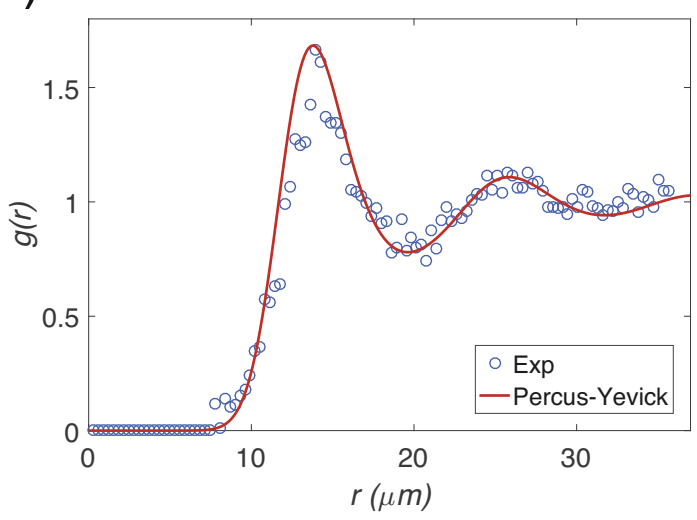

Fig. 1. (a) Two-photon image of a dense fibroblast suspension, field of view of $68 \times 68 \mu \mathrm{m}^{2}$. The average diameter $\mathrm{d}$ of the nearly spherical cells is determined as $d=11.5 \mu \mathrm{m}+/-1.6 \mu \mathrm{m}$ by 3D template-based tracking as described in ref. [12]. At a closer look some hairy protein strings or protrusions can be seen between the spheres. (b) Two-photon image with a larger field of view of $320 \times 320 \mu \mathrm{m}^{2}$. (c) Projections from a z-stack from different points of view. (d) $3 D$ digital reconstruction from tracked cell positions and sizes. ${ }^{[12]}$ In the 3D reconstruction the cells are represented by perfect spherical objects. The measured number density is $4.8 \cdot 10^{5} \mathrm{cell} / \mathrm{s} / \mu \mathrm{l}$. e) Open symbols: radial distribution function of the cell suspension. Comparison to the Percus-Yevick model for hard sphere suspensions: A best fit to the data (solid line) yields a particle diameter of $12.6+/-1.3 \mu \mathrm{m}$ and a volume fraction of $41 \%$. 


\section{Delayed Sedimentation in Cell Suspensions}

We now turn our attention to sedimentation. The sedimentation experiments were carried out in standard cuvettes with a layer thickness of $1 \mathrm{~mm}$ and a volume capacity of $c a .350 \mu \mathrm{l}$. Cuvettes were filled to a height of approximately $2 \mathrm{~cm}$ (Fig. 2). The measurements were carried out using a time lapse camera setup (Leica Digilux 2) with an acquisition rate of one image per 15 minutes. A typical experiment lasts over 20 hours and the typical total number of images recorded is between 80 to 90 . Each time lapse movie is read using a custom-made code which allows us to determine the interface between the supernatant and the sediment by image analysis. The sediment height can be plotted versus the acquisition time, as shown in Fig. 3. The experiments were repeated for different cell concentrations. The lower concentrated suspensions sediment fast at the beginning of the measurements and then come to rest at a constant final height of a weakly compressed sediment. However, if the cell number or initial volume fraction in the suspension is above a certain threshold value of around $10^{5}$ cells $/ \mu$ l the sedimentation proceeds differently. The suspension sediments much more slowly until, at a specific point in time (the collapse time) sedimentation accelerates and the transient gel that was formed collapses as shown in Fig. 3. A closer look at the images in Fig. 2 reveals that the collapse proceeds over the whole volume of the cuvette and the gel first breaks up in domains that become loosely connected eventually leading to failure. When increasing the cell concentration in the suspension the collapse time is increased and also the collapse duration is prolonged (Fig. 3 ). We observe occasionally that the transient gel is stuck at the

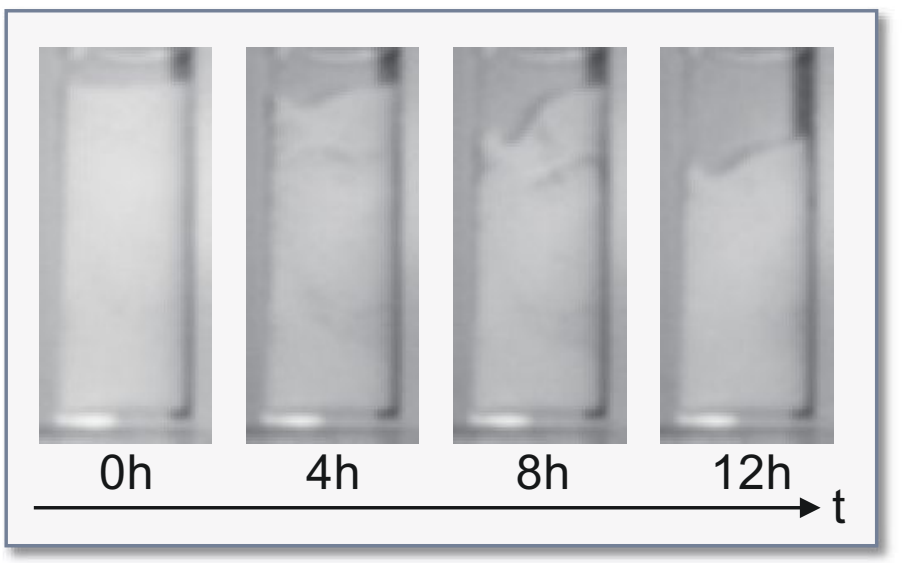

Fig. 2. Sedimentation time lapse images of a cell suspension. The initial concentration is $1.25 \cdot 10^{5}$ cells $/ \mu$ l.

cuvette wall. In such cases the sedimentation process is arrested at a certain height which could be higher than the finite height of a compressed sediment. However, this can be tested by gently shaking that can help to drive the systems towards the final height observed in the absence of wall sticking.

Our experiments suggest that for low concentrations the sticky contacts are not close enough to interact for the transient gel to stabilize and the sedimentation proceeds rapidly. Therefore, the cells sink till they fill the vessel densely. In contrast, for the samples with an increased cell number, the distance between the nearest neighbours is small leading to rapid contact and the formation of weak cell-cell bonds. Thus, a space-filling network, or weak particle gel, can be formed, which does not sediment in the classical sense but coarsens and ages until it finally collapses. Our observations are qualitatively similar to other non-living colloidal systems of attractive colloids, that also show a delayed sedimentation. In the extensive work of the Poon group ${ }^{[18]}$ the attraction between submicron-sized colloidal
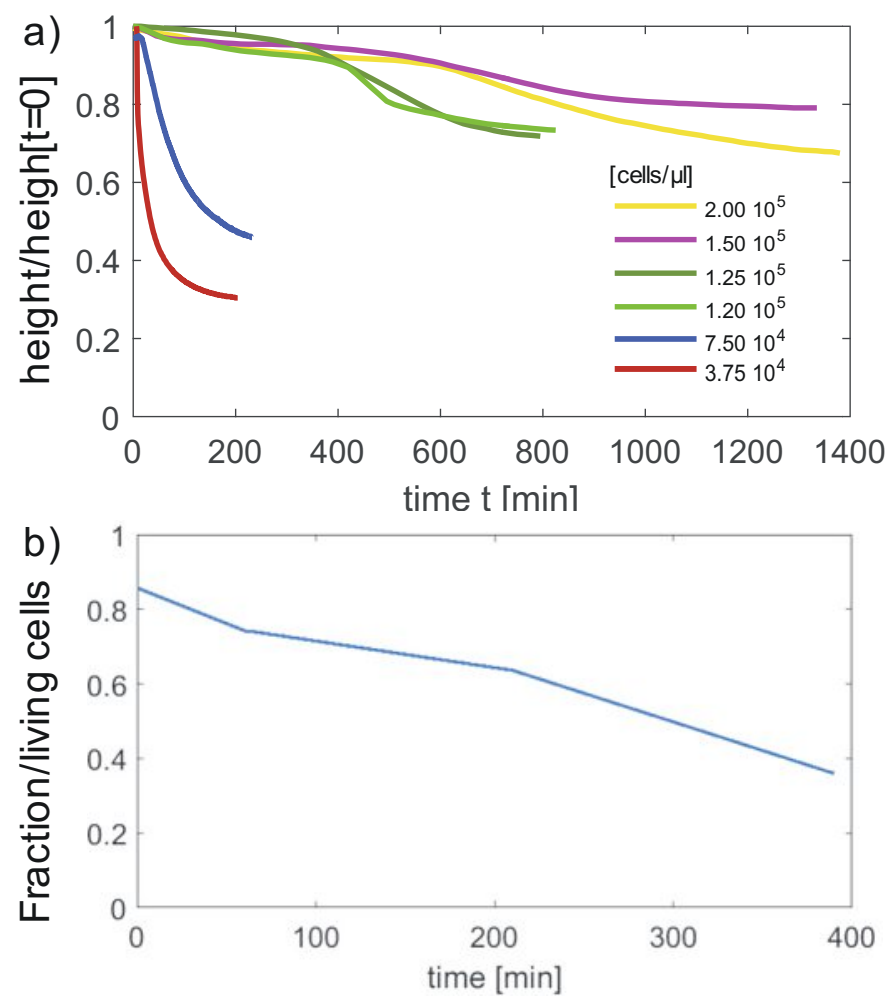

Fig. 3 (a) Sedimentation profile of cell suspensions at different initial concentrations. Note, the lower concentrated specimens sediment rapidly while for higher concentrations a transient gel is formed followed by delayed sedimentation $\left(10^{5} \mathrm{cells} / \mu \mathrm{l} \hat{=} \mathrm{ca}\right.$. $10 \mathrm{vol}$. \%). (b) Evolution of the cell viability over time as determined by labelling the cells with Trypan blue and counting living and dead cells using a Neubauer count chamber.

particles was induced by depletion. The main difference in our work is that the origin of the weak aggregation and gel collapse can be related to the cell attraction and the adhesive surface proteins. The latter is naturally influenced by the ceding viability of the cells (Fig. 3b) but it could for example also be influenced by external triggers such as cell toxins, nutrition and buffer conditions or temperature.

In summary we analysed the structure and sedimentation kinetics of dense assemblies of fibroblast active suspensions by using a two-photon microscope and by monitoring the sedimentation profiles over several hours. In order to increase the cell viability under the two-photon microscope a combination with a lifecell culture chamber has to be considered in future experiments. Within the accuracy of our analysis we cannot detect signatures of attractive interactions in the structure of the cell assembly based on a snapshot of the cell assembly obtained from microscopy. The two-photon microscopy results do however reveal clearly the fuzzy ECM and the protruding ends which are responsible for the attractive interactions between the cells. From our imaging data we can calculate the radial distribution function and the results we obtain are well described by a classical hard-sphere model. While the structure is dominated by classical geometric packing we find a distinct concentration dependence of the sedimentation kinetics. Depending on the initial cell density we observe either rapid or delayed sedimentation. We assume that the origin of this delayed sedimentation is due to attraction mediated by surface proteins leading to the formation a transition gel structure that ages, coarsens and eventually collapses.

Received: Nov. 28, 2018

\section{Acknowledgements}

F.S. would like to thank Justin Cooper-White and Jessica Frith for introducing him to the rheology of NIH 3T3 cell suspensions. This 
work benefitted from financial support by the Swiss National Science Foundation through projects 149867 and 169074 and the National Center of Competence in Research Bio-Inspired Materials. F.S. acknowledges financial support by the Adolphe Merkle Foundation through the Fribourg Center for Nanomaterials and B.R.R. through the Adolphe Merkle Institute.

[1] C. W. Macosko, 'Rheology : principles, measurements, and applications', in 'Advances in interfacial engineering series', 1994, VCH New York, xviii, $550 \mathrm{p}$.

[2] F. Scheffold, F. Cardinaux, T. G. Mason, J. Phys. Cond. Matt. 2013, 25, 50.

[3] P. Fischer, E. J. Windhab, Curr. Opin. Coll. Interf. Sci. 2011, 16, 36.

[4] B. G. C. Maisonneuve, D. C. D. Roux, P. Thorn, J. J. Cooper-White, Biomacromol. 2013, 14, 4388.

[5] F. Scheffold, P. Diaz-Leyva, M. Reufer, J. Harden, Phys. Rev. Lett. 2010, $104,128304$.

[6] T. L. Berezina, S. B. Zaets, C. Morgan, C. R. Spillert, M. Kamiyama, Z. Spolarics, E. A. Deitch, G. W. Machiedo, J. Surgical Res. 2002, 102, 6.

[7] A. Iordan, A. Duperray, C. Verdier, Phys. Rev. E 2008, 77, 011911.

[8] W. Denk, J. H. Strickler, W. W. Webb, Science 1990, 248, 73.

[9] F. Helmchen, W. Denk, Nat. Meth. 2005, 2, 932.
[10] F. Haiss, R. Jolivet, M. T. Wyss, J. Reichold, N. B. Braham, F. Scheffold, M. P. Krafft, B. Weber, J. Physiol. 2009, 587, 3153.

[11] F. Scheffold, 'Principles and fundamentals of optical imaging', in 'Optical Imaging of Neocortical Dynamics', Eds. B. Weber, F. Helmchen, 2014, Springer Humana Press Totowa, NJ, USA.

[12] C. Zhang, C. B. O’Donovan, E. I. Corwin, F. Cardinaux, T. G. Mason, M. E. Möbius, F. Scheffold, Phys. Rev. E 2015, 91, 032302.

[13] J.-P. Hansen, I. R. McDonald, 'Theory of simple liquids', 3rd ed. 2007, Elsevier/Academic Press, Amsterdam Boston.

[14] P. N. Pusey, E. Zaccarelli, C. Valeriani, E. Sanz, E. C. K. Poon, M. E. Cates, Philos. Trans. Roy. Soc. A 2009, 367, 4993.

[15] J. P. Hansen, L. Verlet, Phys. Rev. 1969, 184, 151.

[16] A. Stradner, H. Sedgwick, F. Cardinaux, W. C. Poon, S. U. Egelhaaf, P. Schurtenberger, Nature 2004, 432, 492.

[17] M. S. Wertheim, Phys. Rev. Lett. 1963, 10, 321

[18] A. Jepson, V. A. Martinez, J. Schwarz-Link, W. Poon, Phys. Rev. E 2013, 88, 041002.

[19] J. Schwarz-Linek, C. Valeriani, A. Cacciuto, M. E. Cates, D. Marenduzzo, A. N. Morozov, W. C. K. Poon, Proc. Natl. Acad. Sci. USA 2012, 109, 4052.

[20] W. C. K. Poon, L. Starrs, S. P. Meeker, A. Moussaid, R. M. L. Evans, P. N. Pusey, M. M. Robins, Faraday Disc. 1999, 112, 143.

[21] C. Zhang, G. Brugger, F. Scheffold, Opt. Exp. 2015, 23, 22579. 